\title{
On the Hyers-Ulam Stability of a General Mixed Additive and Cubic Functional Equation in $\boldsymbol{n}$-Banach Spaces
}

\author{
Tian Zhou Xu' ${ }^{1}$ and John Michael Rassias ${ }^{2}$ \\ ${ }^{1}$ School of Mathematics, Beijing Institute of Technology, Beijing 100081, China \\ ${ }^{2}$ Pedagogical Department E.E., Section of Mathematics and Informatics, National and Kapodistrian \\ University of Athens, 4 Agamemnonos Street, Aghia Paraskevi, Athens 15342, Greece
}

Correspondence should be addressed to Tian Zhou Xu, xutianzhou@bit.edu.cn

Received 7 January 2012; Accepted 15 February 2012

Academic Editor: Krzysztof Cieplinski

Copyright (C) 2012 T. Z. Xu and J. M. Rassias. This is an open access article distributed under the Creative Commons Attribution License, which permits unrestricted use, distribution, and reproduction in any medium, provided the original work is properly cited.

The objective of the present paper is to determine the generalized Hyers-Ulam stability of the mixed additive-cubic functional equation in $n$-Banach spaces by the direct method. In addition, we show under some suitable conditions that an approximately mixed additive-cubic function can be approximated by a mixed additive and cubic mapping.

\section{Introduction and Preliminaries}

A basic question in the theory of functional equations is as follows: when is it true that a function, which approximately satisfies a functional equation, must be close to an exact solution of the equation?

If the problem accepts a unique solution, we say the equation is stable (see [1]). The study of stability problems for functional equations is related to a question of Ulam [2] concerning the stability of group homomorphisms and affirmatively answered for Banach spaces by Hyers [3]. The result of Hyers was generalized by Aoki [4] for approximate additive mappings and by Rassias [5] for approximate linear mappings by allowing the Cauchy difference operator $\operatorname{CD} f(x, y)=f(x+y)-[f(x)+f(y)]$ to be controlled by $\epsilon\left(\|x\|^{p}+\|y\|^{p}\right)$. In 1994, a generalization of Rassias' theorem was obtained by Găvruţa [6], who replaced $\epsilon\left(\|x\|^{p}+\|y\|^{p}\right)$ by a general control function $\varphi(x, y)$. On the other hand, several further interesting discussions, modifications, extensions, and generalizations of the original problem of Ulam have been proposed (see, e.g. [7-12] and the references therein).

Recently, Park [9] investigated the approximate additive mappings, approximate Jensen mappings, and approximate quadratic mappings in 2-Banach spaces and proved the 
generalized Hyers-Ulam stability of the Cauchy functional equation, the Jensen functional equation, and the quadratic functional equation in 2-Banach spaces. This is the first result for the stability problem of functional equations in 2-Banach spaces.

In $[11,12]$, we introduced the following mixed additive-cubic functional equation for fixed integers $k$ with $k \neq 0, \pm 1$ :

$$
f(k x+y)+f(k x-y)=k f(x+y)+k f(x-y)+2 f(k x)-2 k f(x),
$$

with $f(0)=0$, and investigated the generalized Hyers-Ulam stability of (1.1) in quasi-Banach spaces and non-Archimedean fuzzy normed spaces, respectively.

In this paper, we investigate, approximate mixed additive-cubic mappings in $n$-Banach spaces. That is, we prove the generalized Hyers-Ulam stability of a general mixed additivecubic equation (1.1) in $n$-Banach spaces by the direct method.

The concept of 2-normed spaces was initially developed by Gähler $[13,14]$ in the middle of 1960s, while that of $n$-normed spaces can be found in $[15,16]$. Since then, many others have studied this concept and obtained various results; see for instance $[15,17-19]$.

We recall some basic facts concerning $n$-normed spaces and some preliminary results.

Definition 1.1. Let $n \in \mathbb{N}$, and let $X$ be a real linear space with $\operatorname{dim} X \geq n$ and $\|\cdot, \ldots, \cdot\|: X^{n} \rightarrow$ $\mathbb{R}$ a function satisfying the following properties:

(N1) $\left\|x_{1}, x_{2}, \ldots, x_{n}\right\|=0$ if and only if $x_{1}, x_{2}, \ldots, x_{n}$ are linearly dependent,

(N2) $\left\|x_{1}, x_{2}, \ldots, x_{n}\right\|$ is invariant under permutation,

(N3) $\left\|\alpha x_{1}, x_{2}, \ldots, x_{n}\right\|=|\alpha|\left\|x_{1}, x_{2}, \ldots, x_{n}\right\|$,

(N4) $\left\|x+y, x_{2}, \ldots, x_{n}\right\| \leq\left\|x, x_{2}, \ldots, x_{n}\right\|+\left\|y, x_{2}, \ldots, x_{n}\right\|$

for all $\alpha \in \mathbb{R}$ and $x, y, x_{1}, x_{2}, \ldots, x_{n} \in \mathrm{X}$. Then the function $\|\cdot \ldots, \cdot\|$ is called an $n$-norm on $\mathrm{X}$ and the pair $(X,\|\cdot, \ldots, \cdot\|)$ is called an $n$-normed space.

Example 1.2. For $x_{1}, x_{2}, \ldots, x_{n} \in \mathbb{R}^{n}$, the Euclidean $n$-norm $\left\|x_{1}, x_{2}, \ldots, x_{n}\right\|_{E}$ is defined by

$$
\left\|x_{1}, x_{2}, \ldots, x_{n}\right\|_{E}=\left|\operatorname{det}\left(x_{i j}\right)\right|=\operatorname{abs}\left(\left|\begin{array}{ccc}
x_{11} & \cdots & x_{1 n} \\
\vdots & \ddots & \vdots \\
x_{n 1} & \cdots & x_{n n}
\end{array}\right|\right)
$$

where $x_{i}=\left(x_{i 1}, \ldots, x_{i n}\right) \in \mathbb{R}^{n}$ for each $i=1,2, \ldots, n$.

Example 1.3. The standard $n$-norm on $X$, a real inner product space of dimension $\operatorname{dim} X \geq n$, is as follows:

$$
\left\|x_{1}, x_{2}, \ldots, x_{n}\right\|_{S}=\left|\begin{array}{ccc}
\left\langle x_{1}, x_{1}\right\rangle & \cdots & \left\langle x_{1}, x_{n}\right\rangle \\
\vdots & \ddots & \vdots \\
\left\langle x_{n}, x_{1}\right\rangle & \cdots & \left\langle x_{n}, x_{n}\right\rangle
\end{array}\right|^{1 / 2}
$$

where $\langle\cdot, \cdot\rangle$ denotes the inner product on $X$. If $X=\mathbb{R}^{n}$, then this $n$-norm is exactly the same as the Euclidean $n$-norm $\left\|x_{1}, x_{2}, \ldots, x_{n}\right\|_{E}$ mentioned earlier. For $n=1$, this $n$-norm is the usual norm $\left\|x_{1}\right\|=\left\langle x_{1}, x_{1}\right\rangle^{1 / 2}$. 
Definition 1.4. A sequence $\left\{x_{k}\right\}$ in an $n$-normed space $X$ is said to converge to some $x \in X$ in the $n$-norm if

$$
\lim _{k \rightarrow \infty}\left\|x_{k}-x, y_{2}, \ldots, y_{n}\right\|=0
$$

for every $y_{2}, \ldots, y_{n} \in X$.

Definition 1.5. A sequence $\left\{x_{k}\right\}$ in an $n$-normed space $X$ is said to be a Cauchy sequence with respect to the $n$-norm if

$$
\lim _{k, l \rightarrow \infty}\left\|x_{k}-x_{l}, y_{2}, \ldots, y_{n}\right\|=0
$$

for every $y_{2}, \ldots, y_{n} \in X$. If every Cauchy sequence in $X$ converges to some $x \in X$, then $X$ is said to be complete with respect to the $n$-norm. Any complete $n$-normed space is said to be an $n$-Banach space.

Now we state the following results as lemma (see [9] for the details).

Lemma 1.6. Let $X$ be an n-normed space. Then,

(1) For $x_{i} \in X(i=1, \ldots, n)$ and $\gamma$, a real number,

$$
\left\|x_{1}, \ldots, x_{i}, \ldots, x_{j}, \ldots, x_{n}\right\|=\left\|x_{1}, \ldots, x_{i}, \ldots, x_{j}+\gamma x_{i}, \ldots, x_{n}\right\|
$$

for all $1 \leq i \neq j \leq n$,

(2) $\left|\left\|x, y_{2}, \ldots, y_{n}\right\|-\left\|y, y_{2}, \ldots, y_{n}\right\|\right| \leq\left\|x-y, y_{2}, \ldots, y_{n}\right\|$ for all $x, y, y_{2}, \ldots, y_{n} \in X$,

(3) if $\left\|x, y_{2}, \ldots, y_{n}\right\|=0$ for all $y_{2}, \ldots, y_{n} \in X$, then $x=0$,

(4) for a convergent sequence $\left\{x_{j}\right\}$ in $X$,

$$
\lim _{j \rightarrow \infty}\left\|x_{j}, y_{2}, \ldots, y_{n}\right\|=\left\|\lim _{j \rightarrow \infty} x_{j}, y_{2}, \ldots, y_{n}\right\|
$$

for all $y_{2}, \ldots, y_{n} \in X$.

\section{Approximate Mixed Additive-Cubic Mappings}

In this section, we investigate the generalized Hyers-Ulam stability of the generalized mixed additive-cubic functional equation in $n$-Banach spaces. Let $X$ be a linear space and $Y$ an $n$ Banach space. For convenience, we use the following abbreviation for a given mapping $f$ : $X \rightarrow Y$ :

$$
D f(x, y):=f(k x+y)+f(k x-y)-k f(x+y)-k f(x-y)-2 f(k x)+2 k f(x)
$$

for all $x, y \in X$. 
Theorem 2.1. Let $X$ be a linear space and $Y$ an $n$-Banach space. Let $f: X \rightarrow Y$ be a mapping with $f(0)=0$ for which there is a function $\varphi: X^{n+1} \rightarrow[0, \infty)$ such that

$$
\begin{gathered}
\sum_{j=0}^{\infty} \frac{1}{2^{j}} \varphi\left(2^{j} x, 2^{j} y, u_{2}, \ldots, u_{n}\right)<\infty, \\
\left\|D f(x, y), u_{2}, \ldots, u_{n}\right\|_{Y} \leq \varphi\left(x, y, u_{2}, \ldots, u_{n}\right)
\end{gathered}
$$

for all $x, y, u_{2}, \ldots, u_{n} \in X$. Then, there is a unique additive mapping $A: X \rightarrow Y$ such that

$$
\left\|f(2 x)-8 f(x)-A(x), u_{2}, \ldots, u_{n}\right\|_{Y} \leq \sum_{j=0}^{\infty} \frac{1}{2^{j+1}} \tilde{\varphi}\left(2^{j} x, u_{2}, \ldots, u_{n}\right)
$$

for all $x, u_{2}, \ldots, u_{n} \in X$, where

$$
\begin{aligned}
\tilde{\varphi}\left(x, u_{2}, \ldots, u_{n}\right) & \\
:=\frac{1}{\left|k^{3}-k\right|}\{ & (|k|+1)\left[\varphi\left(x,(2 k+1) x, u_{2}, \ldots, u_{n}\right)+\varphi\left(x,(2 k-1) x, u_{2}, \ldots, u_{n}\right)\right] \\
& +\varphi\left(3 x, x, u_{2}, \ldots, u_{n}\right)+\left(8 k^{2}+1\right) \varphi\left(x, x, u_{2}, \ldots, u_{n}\right)+\varphi\left(x, 3 k x, u_{2}, \ldots, u_{n}\right) \\
& +\varphi\left(x, k x, u_{2}, \ldots, u_{n}\right)+k^{2} \varphi\left(2 x, 2 x, u_{2}, \ldots, u_{n}\right)+\varphi\left(2 x, 2 k x, u_{2}, \ldots, u_{n}\right) \\
& +2 \varphi\left(x,(k+1) x, u_{2}, \ldots, u_{n}\right)+2 \varphi\left(x,(k-1) x, u_{2}, \ldots, u_{n}\right)+2 \varphi\left(2 x, x, u_{2}, \ldots, u_{n}\right) \\
& +2 \varphi\left(2 x, k x, u_{2}, \ldots, u_{n}\right)+8 \varphi\left(\frac{x}{2}, \frac{k x}{2}, u_{2}, \ldots, u_{n}\right) \\
& +8|k| \varphi\left(\frac{x}{2}, \frac{(2 k-1) x}{2}, u_{2}, \ldots, u_{n}\right)+8|k| \varphi\left(\frac{x}{2}, \frac{(2 k+1) x}{2}, u_{2}, \ldots, u_{n}\right) \\
& +8 \varphi\left(\frac{x}{2}, \frac{3 k x}{2}, u_{2}, \ldots, u_{n}\right)+\frac{|k|+1}{|k-1|} \varphi\left(0,(k+1) x, u_{2}, \ldots, u_{n}\right) \\
& +\frac{8 k^{2}+1}{|k-1|} \varphi\left(0,(k-1) x, u_{2}, \ldots, u_{n}\right)+\frac{2}{|k-1|} \varphi\left(0, x, u_{2}, \ldots, u_{n}\right) \\
& +\frac{|k|}{|k-1|} \varphi\left(0,(3 k-1) x, u_{2}, \ldots, u_{n}\right)+\frac{k^{2}}{|k-1|} \varphi\left(0,2(k-1) x, u_{2}, \ldots, u_{n}\right) \\
& +\frac{k^{2}+|k|-1}{|k-1|} \varphi\left(0,2 k x, u_{2}, \ldots, u_{n}\right) \\
& +\frac{8|k|}{|k-1|} \varphi\left(0, \frac{(3 k-1) x}{2}, u_{2}, \ldots, u_{n}\right)+\frac{8|k|}{|k-1|} \varphi\left(0, \frac{(k+1) x}{2}, u_{2}, \ldots, u_{n}\right) \\
& \left.+\frac{8 k^{2}+2|k|-8}{|k-1|} \varphi\left(0, k x, u_{2}, \ldots, u_{n}\right)\right\} . \\
& =2(2,5)
\end{aligned}
$$


Proof. Letting $x=0$ in (2.3), we get

$$
\left\|f(y)+f(-y), u_{2}, \ldots, u_{n}\right\|_{Y} \leq \frac{1}{|k-1|} \varphi\left(0, y, u_{2}, \ldots, u_{n}\right)
$$

for all $y, u_{2}, \ldots, u_{n} \in X$. Putting $y=x$ in (2.3), we have

$$
\left\|f((k+1) x)+f((k-1) x)-k f(2 x)-2 f(k x)+2 k f(x), u_{2}, \ldots, u_{n}\right\|_{Y} \leq \varphi\left(x, x, u_{2}, \ldots, u_{n}\right)
$$

for all $x, u_{2}, \ldots, u_{n} \in X$. Thus

$$
\begin{aligned}
& \left\|f(2(k+1) x)+f(2(k-1) x)-k f(4 x)-2 f(2 k x)+2 k f(2 x), u_{2}, \ldots, u_{n}\right\|_{Y} \\
& \quad \leq \varphi\left(2 x, 2 x, u_{2}, \ldots, u_{n}\right)
\end{aligned}
$$

for all $x, u_{2}, \ldots, u_{n} \in X$. Letting $y=k x$ in (2.3), we get

$\left\|f(2 k x)-k f((k+1) x)-k f(-(k-1) x)-2 f(k x)+2 k f(x), u_{2}, \ldots, u_{n}\right\|_{Y} \leq \varphi\left(x, k x, u_{2}, \ldots, u_{n}\right)$

for all $x, u_{2}, \ldots, u_{n} \in X$. Letting $y=(k+1) x$ in (2.3), we have

$$
\begin{aligned}
& \left\|f((2 k+1) x)+f(-x)-k f((k+2) x)-k f(-k x)-2 f(k x)+2 k f(x), u_{2}, \ldots, u_{n}\right\|_{Y} \\
& \quad \leq \varphi\left(x,(k+1) x, u_{2}, \ldots, u_{n}\right)
\end{aligned}
$$

for all $x, u_{2}, \ldots, u_{n} \in X$. Letting $y=(k-1) x$ in (2.3), we have

$$
\begin{aligned}
& \left\|f((2 k-1) x)-(k+2) f(k x)-k f(-(k-2) x)+(2 k+1) f(x), u_{2}, \ldots, u_{n}\right\|_{Y} \\
& \quad \leq \varphi\left(x,(k-1) x, u_{2}, \ldots, u_{n}\right)
\end{aligned}
$$

for all $x, u_{2}, \ldots, u_{n} \in X$. Replacing $x$ and $y$ by $2 x$ and $x$ in (2.3), respectively, we get

$$
\begin{aligned}
& \left\|f((2 k+1) x)+f((2 k-1) x)-2 f(2 k x)-k f(3 x)+2 k f(2 x)-k f(x), u_{2}, \ldots, u_{n}\right\|_{Y} \\
& \quad \leq \varphi\left(2 x, x, u_{2}, \ldots, u_{n}\right)
\end{aligned}
$$

for all $x, u_{2}, \ldots, u_{n} \in X$. Replacing $x$ and $y$ by $3 x$ and $x$ in (2.3), respectively, we get

$$
\begin{aligned}
& \left\|f((3 k+1) x)+f((3 k-1) x)-2 f(3 k x)-k f(4 x)-k f(2 x)+2 k f(3 x), u_{2}, \ldots, u_{n}\right\|_{Y} \\
& \quad \leq \varphi\left(3 x, x, u_{2}, \ldots, u_{n}\right)
\end{aligned}
$$


for all $x, u_{2}, \ldots, u_{n} \in X$. Replacing $x$ and $y$ by $2 x$ and $k x$ in (2.3), respectively, we have

$$
\begin{aligned}
& \left\|f(3 k x)+f(k x)-k f((k+2) x)-k f(-(k-2) x)-2 f(2 k x)+2 k f(2 x), u_{2}, \ldots, u_{n}\right\|_{Y} \\
& \quad \leq \varphi\left(2 x, k x, u_{2}, \ldots, u_{n}\right)
\end{aligned}
$$

for all $x, u_{2}, \ldots, u_{n} \in X$. Setting $y=(2 k+1) x$ in (2.3), we have

$$
\begin{aligned}
& \left\|f((3 k+1) x)+f(-(k+1) x)-k f(2(k+1) x)-k f(-2 k x)-2 f(k x)+2 k f(x), u_{2}, \ldots, u_{n}\right\|_{Y} \\
& \quad \leq \varphi\left(x,(2 k+1) x, u_{2}, \ldots, u_{n}\right)
\end{aligned}
$$

for all $x, u_{2}, \ldots, u_{n} \in X$. Letting $y=(2 k-1) x$ in (2.3), we have

$$
\begin{aligned}
& \left\|f((3 k-1) x)+f(-(k-1) x)-k f(-2(k-1) x)-k f(2 k x)-2 f(k x)+2 k f(x), u_{2}, \ldots, u_{n}\right\|_{Y} \\
& \quad \leq \varphi\left(x,(2 k-1) x, u_{2}, \ldots, u_{n}\right)
\end{aligned}
$$

for all $x, u_{2}, \ldots, u_{n} \in X$. Letting $y=3 k x$ in (2.3), we have

$$
\begin{aligned}
& \left\|f(4 k x)+f(-2 k x)-k f((3 k+1) x)-k f(-(3 k-1) x)-2 f(k x)+2 k f(x), u_{2}, \ldots, u_{n}\right\|_{Y} \\
& \quad \leq \varphi\left(x, 3 k x, u_{2}, \ldots, u_{n}\right)
\end{aligned}
$$

for all $x, u_{2}, \ldots, u_{n} \in X$. By (2.6), (2.7), (2.13), (2.15), and (2.16), we get

$$
\begin{aligned}
&\left\|k f(2(k+1) x)+k f(-2(k-1) x)+6 f(k x)-2 f(3 k x)-k f(4 x)+2 k f(3 x)-6 k f(x), u_{2}, \ldots, u_{n}\right\|_{Y} \\
& \leq \varphi\left(x,(2 k+1) x, u_{2}, \ldots, u_{n}\right)+\varphi\left(x,(2 k-1) x, u_{2}, \ldots, u_{n}\right)+\varphi\left(3 x, x, u_{2}, \ldots, u_{n}\right) \\
&+\varphi\left(x, x, u_{2}, \ldots, u_{n}\right)+\frac{1}{|k-1|} \varphi\left(0,(k+1) x, u_{2}, \ldots, u_{n}\right) \\
& \quad+\frac{1}{|k-1|} \varphi\left(0,(k-1) x, u_{2}, \ldots, u_{n}\right)+\frac{|k|}{|k-1|} \varphi\left(0,2 k x, u_{2}, \ldots, u_{n}\right)
\end{aligned}
$$

for all $x, u_{2}, \ldots, u_{n} \in X$. By (2.6), (2.10), and (2.11), we have

$$
\begin{aligned}
& \left\|f((2 k+1) x)+f((2 k-1) x)-k f((k+2) x)-k f(-(k-2) x)-4 f(k x)+4 k f(x), u_{2}, \ldots, u_{n}\right\|_{Y} \\
& \leq \varphi\left(x,(k+1) x, u_{2}, \ldots, u_{n}\right)+\varphi\left(x,(k-1) x, u_{2}, \ldots, u_{n}\right)+\frac{1}{|k-1|} \varphi\left(0, x, u_{2}, \ldots, u_{n}\right) \\
& \quad+\left|\frac{k}{k-1}\right| \varphi\left(0, k x, u_{2}, \ldots, u_{n}\right)
\end{aligned}
$$


for all $x, u_{2}, \ldots, u_{n} \in X$. It follows from (2.12) and (2.19) that

$$
\begin{aligned}
& \left\|k f((k+2) x)+k f(-(k-2) x)-2 f(2 k x)+4 f(k x)-k f(3 x)+2 k f(2 x)-5 k f(x), u_{2}, \ldots, u_{n}\right\|_{Y} \\
& \leq \varphi\left(x,(k+1) x, u_{2}, \ldots, u_{n}\right)+\varphi\left(x,(k-1) x, u_{2}, \ldots, u_{n}\right)+\varphi\left(2 x, x, u_{2}, \ldots, u_{n}\right) \\
& \quad+\frac{1}{|k-1|} \varphi\left(0, x, u_{2}, \ldots, u_{n}\right)+\left|\frac{k}{k-1}\right| \varphi\left(0, k x, u_{2}, \ldots, u_{n}\right)
\end{aligned}
$$

for all $x, u_{2}, \ldots, u_{n} \in X$. By (2.14) and (2.20), we have

$$
\begin{aligned}
& \left\|f(3 k x)-4 f(2 k x)+5 f(k x)-k f(3 x)+4 k f(2 x)-5 k f(x), u_{2}, \ldots, u_{n}\right\|_{Y} \\
& \leq \varphi\left(x,(k+1) x, u_{2}, \ldots, u_{n}\right)+\varphi\left(x,(k-1) x, u_{2}, \ldots, u_{n}\right)+\varphi\left(2 x, x, u_{2}, \ldots, u_{n}\right) \\
& \quad+\varphi\left(2 x, k x, u_{2}, \ldots, u_{n}\right)+\frac{1}{|k-1|} \varphi\left(0, x, u_{2}, \ldots, u_{n}\right)+\left|\frac{k}{k-1}\right| \varphi\left(0, k x, u_{2}, \ldots, u_{n}\right)
\end{aligned}
$$

for all $x, u_{2}, \ldots, u_{n} \in X$. By (2.6), (2.15), (2.16), and (2.17), we have

$$
\begin{aligned}
& \| k f(-(k+1) x)-k f(-(k-1) x)-k^{2} f(2(k+1) x)+k^{2} f(-2(k-1) x) \\
& \quad+k^{2} f(2 k x)-\left(k^{2}-1\right) f(-2 k x)+f(4 k x)-2 f(k x)+2 k f(x), u_{2}, \ldots, u_{n} \|_{Y} \\
& \leq|k| \varphi\left(x,(2 k+1) x, u_{2}, \ldots, u_{n}\right)+|k| \varphi\left(x,(2 k-1) x, u_{2}, \ldots, u_{n}\right)+\varphi\left(x, 3 k x, u_{2}, \ldots, u_{n}\right) \\
& \quad+\left|\frac{k}{k-1}\right| \varphi\left(0,(3 k-1) x, u_{2}, \ldots, u_{n}\right)
\end{aligned}
$$

for all $x, u_{2}, \ldots, u_{n} \in X$. It follows from (2.6), (2.8), (2.9), and (2.22) that

$$
\begin{aligned}
&\left\|f(4 k x)-2 f(2 k x)-k^{3} f(4 x)+2 k^{3} f(2 x), u_{2}, \ldots, u_{n}\right\|_{Y} \\
& \leq \mid k\left|\varphi\left(x,(2 k+1) x, u_{2}, \ldots, u_{n}\right)+\right| k \mid \varphi\left(x,(2 k-1) x, u_{2}, \ldots, u_{n}\right)+\varphi\left(x, 3 k x, u_{2}, \ldots, u_{n}\right) \\
&+\varphi\left(x, k x, u_{2}, \ldots, u_{n}\right)+k^{2} \varphi\left(2 x, 2 x, u_{2}, \ldots, u_{n}\right)+\left|\frac{k}{k-1}\right| \varphi\left(0,(3 k-1) x, u_{2}, \ldots, u_{n}\right) \\
&+\left|\frac{k}{k-1}\right| \varphi\left(0,(k+1) x, u_{2}, \ldots, u_{n}\right)+\frac{k^{2}}{|k-1|} \varphi\left(0,2(k-1) x, u_{2}, \ldots, u_{n}\right) \\
&+\frac{k^{2}-1}{|k-1|} \varphi\left(0,2 k x, u_{2}, \ldots, u_{n}\right)
\end{aligned}
$$


for all $x, u_{2}, \ldots, u_{n} \in X$. Hence,

$$
\begin{aligned}
&\left\|f(2 k x)-2 f(k x)-k^{3} f(2 x)+2 k^{3} f(x), u_{2}, \ldots, u_{n}\right\|_{Y} \\
& \leq \mid k\left|\varphi\left(\frac{x}{2}, \frac{(2 k+1) x}{2}, u_{2}, \ldots, u_{n}\right)+\right| k \mid \varphi\left(\frac{x}{2}, \frac{(2 k-1) x}{2}, u_{2}, \ldots, u_{n}\right)+\varphi\left(\frac{x}{2}, \frac{3 k x}{2}, u_{2}, \ldots, u_{n}\right) \\
&+\varphi\left(\frac{x}{2}, \frac{k x}{2}, u_{2}, \ldots, u_{n}\right)+k^{2} \varphi\left(x, x, u_{2}, \ldots, u_{n}\right)+\left|\frac{k}{k-1}\right| \varphi\left(0, \frac{(3 k-1) x}{2}, u_{2}, \ldots, u_{n}\right) \\
&+\left|\frac{k}{k-1}\right| \varphi\left(0, \frac{(k+1) x}{2}, u_{2}, \ldots, u_{n}\right)+\frac{k^{2}}{|k-1|} \varphi\left(0,(k-1) x, u_{2}, \ldots, u_{n}\right) \\
&+\frac{k^{2}-1}{|k-1|} \varphi\left(0, k x, u_{2}, \ldots, u_{n}\right)
\end{aligned}
$$

for all $x, u_{2}, \ldots, u_{n} \in X$. By (2.9), we have

$$
\begin{aligned}
& \left\|f(4 k x)-k f(2(k+1) x)-k f(-2(k-1) x)-2 f(2 k x)+2 k f(2 x), u_{2}, \ldots, u_{n}\right\|_{Y} \\
& \quad \leq \varphi\left(2 x, 2 k x, u_{2}, \ldots, u_{n}\right)
\end{aligned}
$$

for all $x, u_{2}, \ldots, u_{n} \in X$. From (2.23) and (2.25), we have

$$
\begin{aligned}
&\left\|k f(2(k+1) x)+k f(-2(k-1) x)-k^{3} f(4 x)+\left(2 k^{3}-2 k\right) f(2 x)\right\|_{\Upsilon} \\
& \leq|k| \varphi\left(x,(2 k+1) x, u_{2}, \ldots, u_{n}\right)+|k| \varphi\left(x,(2 k-1) x, u_{2}, \ldots, u_{n}\right)+\varphi\left(x, 3 k x, u_{2}, \ldots, u_{n}\right) \\
&+\varphi\left(x, k x, u_{2}, \ldots, u_{n}\right)+k^{2} \varphi\left(2 x, 2 x, u_{2}, \ldots, u_{n}\right)+\varphi\left(2 x, 2 k x, u_{2}, \ldots, u_{n}\right) \\
&+\left|\frac{k}{k-1}\right| \varphi\left(0,(3 k-1) x, u_{2}, \ldots, u_{n}\right)+\left|\frac{k}{k-1}\right| \varphi\left(0,(k+1) x, u_{2}, \ldots, u_{n}\right) \\
&+\frac{k^{2}}{|k-1|} \varphi\left(0,2(k-1) x, u_{2}, \ldots, u_{n}\right)+\frac{k^{2}-1}{|k-1|} \varphi\left(0,2 k x, u_{2}, \ldots, u_{n}\right)
\end{aligned}
$$

for all $x, u_{2}, \ldots, u_{n} \in X$. Also, from (2.18) and (2.26), we get

$$
\begin{aligned}
&\left\|2 f(3 k x)-6 f(k x)+\left(k-k^{3}\right) f(4 x)-2 k f(3 x)+\left(2 k^{3}-2 k\right) f(2 x)+6 k f(x), u_{2}, \ldots, u_{n}\right\|_{Y} \\
& \leq(|k|+1)\left[\varphi\left(x,(2 k+1) x, u_{2}, \ldots, u_{n}\right)+\varphi\left(x,(2 k-1) x, u_{2}, \ldots, u_{n}\right)\right]+\varphi\left(3 x, x, u_{2}, \ldots, u_{n}\right) \\
&+\varphi\left(x, x, u_{2}, \ldots, u_{n}\right)+\varphi\left(x, 3 k x, u_{2}, \ldots, u_{n}\right)+\varphi\left(x, k x, u_{2}, \ldots, u_{n}\right) \\
&+k^{2} \varphi\left(2 x, 2 x, u_{2}, \ldots, u_{n}\right)+\varphi\left(2 x, 2 k x, u_{2}, \ldots, u_{n}\right)+\frac{|k|+1}{|k-1|} \varphi\left(0,(k+1) x, u_{2}, \ldots, u_{n}\right)
\end{aligned}
$$


Abstract and Applied Analysis

$$
\begin{aligned}
& +\frac{1}{|k-1|} \varphi\left(0,(k-1) x, u_{2}, \ldots, u_{n}\right)+\frac{k^{2}+|k|-1}{|k-1|} \varphi\left(0,2 k x, u_{2}, \ldots, u_{n}\right) \\
& +\left|\frac{k}{k-1}\right| \varphi\left(0,(3 k-1) x, u_{2}, \ldots, u_{n}\right)+\frac{k^{2}}{|k-1|} \varphi\left(0,2(k-1) x, u_{2}, \ldots, u_{n}\right)
\end{aligned}
$$

for all $x, u_{2}, \ldots, u_{n} \in X$.

On the other hand, it follows from (2.21) and (2.27) that

$$
\begin{aligned}
\| 8 f( & (k x)-16 f(k x)+\left(k-k^{3}\right) f(4 x)+\left(2 k^{3}-10 k\right) f(2 x)+16 k f(x), u_{2}, \ldots, u_{n} \|_{Y} \\
\leq & (|k|+1)\left[\varphi\left(x,(2 k+1) x, u_{2}, \ldots, u_{n}\right)+\varphi\left(x,(2 k-1) x, u_{2}, \ldots, u_{n}\right)\right]+\varphi\left(3 x, x, u_{2}, \ldots, u_{n}\right) \\
& +\varphi\left(x, x, u_{2}, \ldots, u_{n}\right)+\varphi\left(x, 3 k x, u_{2}, \ldots, u_{n}\right)+\varphi\left(x, k x, u_{2}, \ldots, u_{n}\right) \\
& +k^{2} \varphi\left(2 x, 2 x, u_{2}, \ldots, u_{n}\right)+\varphi\left(2 x, 2 k x, u_{2}, \ldots, u_{n}\right)+2 \varphi\left(x,(k+1) x, u_{2}, \ldots, u_{n}\right) \\
& +2 \varphi\left(x,(k-1) x, u_{2}, \ldots, u_{n}\right)+2 \varphi\left(2 x, x, u_{2}, \ldots, u_{n}\right)+2 \varphi\left(2 x, k x, u_{2}, \ldots, u_{n}\right) \\
& +\frac{2}{|k-1|} \varphi\left(0, x, u_{2}, \ldots, u_{n}\right)+\frac{2|k|}{|k-1|} \varphi\left(0, k x, u_{2}, \ldots, u_{n}\right)+\frac{|k|+1}{|k-1|} \varphi\left(0,(k+1) x, u_{2}, \ldots, u_{n}\right) \\
& +\frac{1}{|k-1|} \varphi\left(0,(k-1) x, u_{2}, \ldots, u_{n}\right)+\frac{k^{2}+|k|-1}{|k-1|} \varphi\left(0,2 k x, u_{2}, \ldots, u_{n}\right) \\
& +\left|\frac{k}{k-1}\right| \varphi\left(0,(3 k-1) x, u_{2}, \ldots, u_{n}\right)+\frac{k^{2}}{|k-1|} \varphi\left(0,2(k-1) x, u_{2}, \ldots, u_{n}\right)
\end{aligned}
$$

for all $x, u_{2}, \ldots, u_{n} \in X$. Therefore by (2.24) and (2.28), we get

$$
\begin{aligned}
\| f(4 x) & -10 f(2 x)+16 f(x), u_{2}, \ldots, u_{n} \|_{Y} \\
\leq & \frac{1}{\left|k^{3}-k\right|} \\
\quad \times & \left\{(|k|+1)\left[\varphi\left(x,(2 k+1) x, u_{2}, \ldots, u_{n}\right)+\varphi\left(x,(2 k-1) x, u_{2}, \ldots, u_{n}\right)\right]\right. \\
& +\varphi\left(3 x, x, u_{2}, \ldots, u_{n}\right)+\left(8 k^{2}+1\right) \varphi\left(x, x, u_{2}, \ldots, u_{n}\right)+\varphi\left(x, 3 k x, u_{2}, \ldots, u_{n}\right) \\
& +\varphi\left(x, k x, u_{2}, \ldots, u_{n}\right)+k^{2} \varphi\left(2 x, 2 x, u_{2}, \ldots, u_{n}\right)+\varphi\left(2 x, 2 k x, u_{2}, \ldots, u_{n}\right) \\
& +2 \varphi\left(x,(k+1) x, u_{2}, \ldots, u_{n}\right)+2 \varphi\left(x,(k-1) x, u_{2}, \ldots, u_{n}\right)+2 \varphi\left(2 x, x, u_{2}, \ldots, u_{n}\right) \\
& +2 \varphi\left(2 x, k x, u_{2}, \ldots, u_{n}\right)+8 \varphi\left(\frac{x}{2}, \frac{k x}{2}, u_{2}, \ldots, u_{n}\right)+8|k| \varphi\left(\frac{x}{2}, \frac{(2 k-1) x}{2}, u_{2}, \ldots, u_{n}\right) \\
& +8|k| \varphi\left(\frac{x}{2}, \frac{(2 k+1) x}{2}, u_{2}, \ldots, u_{n}\right)+8 \varphi\left(\frac{x}{2}, \frac{3 k x}{2}, u_{2}, \ldots, u_{n}\right)
\end{aligned}
$$




$$
\begin{aligned}
& +\frac{|k|+1}{|k-1|} \varphi\left(0,(k+1) x, u_{2}, \ldots, u_{n}\right)+\frac{8 k^{2}+1}{|k-1|} \varphi\left(0,(k-1) x, u_{2}, \ldots, u_{n}\right) \\
& +\frac{2}{|k-1|} \varphi\left(0, x, u_{2}, \ldots, u_{n}\right)+\left|\frac{k}{k-1}\right| \varphi\left(0,(3 k-1) x, u_{2}, \ldots, u_{n}\right) \\
& +\frac{k^{2}}{|k-1|} \varphi\left(0,2(k-1) x, u_{2}, \ldots, u_{n}\right)+\frac{k^{2}+|k|-1}{|k-1|} \varphi\left(0,2 k x, u_{2}, \ldots, u_{n}\right) \\
& +\frac{8|k|}{|k-1|} \varphi\left(0, \frac{(3 k-1) x}{2}, u_{2}, \ldots, u_{n}\right) \\
& \left.+\frac{8|k|}{|k-1|} \varphi\left(0, \frac{(k+1) x}{2}, u_{2}, \ldots, u_{n}\right)+\frac{8 k^{2}+2|k|-8}{|k-1|} \varphi\left(0, k x, u_{2}, \ldots, u_{n}\right)\right\} \\
& :=\tilde{\varphi}\left(x, u_{2}, \ldots, u_{n}\right)
\end{aligned}
$$

for all $x, u_{2}, \ldots, u_{n} \in X$.

Now, let $g: X \rightarrow Y$ be the mapping defined by $g(x):=f(2 x)-8 f(x)$ for all $x, u_{2}, \ldots, u_{n} \in X$. Then, (2.29) means that

$$
\left\|f(4 x)-10 f(2 x)+16 f(x), u_{2}, \ldots, u_{n}\right\|_{Y} \leq \tilde{\varphi}\left(x, u_{2}, \ldots, u_{n}\right)
$$

for all $x, u_{2}, \ldots, u_{n} \in X$. Also, we get

$$
\left\|g(2 x)-2 g(x), u_{2}, \ldots, u_{n}\right\|_{Y} \leq \tilde{\varphi}\left(x, u_{2}, \ldots, u_{n}\right)
$$

for all $x \in X$. Replacing $x$ by $2^{j} x$ in (2.31) and dividing both sides of (2.31) by $2^{j+1}$, we get

$$
\left\|\frac{1}{2^{j}} g\left(2^{j} x\right)-\frac{1}{2^{j+1}} g\left(2^{j+1} x\right), u_{2}, \ldots, u_{n}\right\|_{Y} \leq \frac{1}{2^{j+1}} \tilde{\varphi}\left(2^{j} x, u_{2}, \ldots, u_{n}\right)
$$

for all $x, u_{2}, \ldots, u_{n} \in X$ and all integers $j \geq 0$. For all integers $l, m$ with $0 \leq l<m$, we have

$$
\begin{aligned}
\left\|\frac{1}{2^{l}} g\left(2^{l} x\right)-\frac{1}{2^{m}} g\left(2^{m} x\right), u_{2}, \ldots, u_{n}\right\|_{Y} & \leq \sum_{j=l}^{m-1}\left\|\frac{1}{2^{j}} g\left(2^{j} x\right)-\frac{1}{2^{j+1}} g\left(2^{j+1} x\right), u_{2}, \ldots, u_{n}\right\|_{Y} \\
& \leq \sum_{j=l}^{m-1} \frac{1}{2^{j+1}} \tilde{\varphi}\left(2^{j} x, u_{2}, \ldots, u_{n}\right)
\end{aligned}
$$

for all $x, u_{2}, \ldots, u_{n} \in X$. So, we get

$$
\lim _{l, m \rightarrow \infty}\left\|\frac{1}{2^{l}} g\left(2^{l} x\right)-\frac{1}{2^{m}} g\left(2^{m} x\right), u_{2}, \ldots, u_{n}\right\|_{Y}=0
$$


for all $x, u_{2}, \ldots, u_{n} \in X$. This shows that the sequence $\left\{\left(1 / 2^{j}\right) g\left(2^{j} x\right)\right\}$ is a Cauchy sequence in $Y$. Since $Y$ is an $n$-Banach space, the sequence $\left\{\left(1 / 2^{j}\right) g\left(2^{j} x\right)\right\}$ converges. So, we can define a mapping $A: X \rightarrow Y$ by

$$
A(x):=\lim _{j \rightarrow \infty} \frac{1}{2^{j}} g\left(2^{j} x\right)
$$

for all $x \in X$. Putting $l=0$, then passing the limit $m \rightarrow \infty$ in (2.33), and using Lemma 1.6(4), we get

$$
\left\|g(x)-A(x), u_{2}, \ldots, u_{n}\right\|_{Y} \leq \sum_{j=0}^{\infty} \frac{1}{2^{j+1}} \tilde{\varphi}\left(2^{j} x, u_{2}, \ldots, u_{n}\right)
$$

for all $x, u_{2}, \ldots, u_{n} \in X$.

Now we show that $A$ is additive. By Lemma 1.6, (2.2), (2.32), and (2.35), we have

$$
\begin{aligned}
\left\|A(2 x)-2 A(x), u_{2}, \ldots, u_{n}\right\|_{Y} & =\lim _{j \rightarrow \infty}\left\|\frac{1}{2^{j}} g\left(2^{j+1} x\right)-\frac{1}{2^{j-1}} g\left(2^{j} x\right), u_{2}, \ldots, u_{n}\right\|_{Y} \\
& =2 \lim _{j \rightarrow \infty}\left\|\frac{1}{2^{j+1}} g\left(2^{j+1} x\right)-\frac{1}{2^{j}} g\left(2^{j} x\right), u_{2}, \ldots, u_{n}\right\|_{Y} \\
& \leq \lim _{j \rightarrow \infty} \frac{1}{2^{j}} \tilde{\varphi}\left(2^{j} x, u_{2}, \ldots, u_{n}\right)=0
\end{aligned}
$$

for all $x, u_{2}, \ldots, u_{n} \in X$. By Lemma 1.6(3), $A(2 x)=2 A(x)$ for all $x \in X$. Also, by Lemma 1.6(4), (2.2), (2.3), and (2.35), we get

$$
\begin{array}{rl}
\| D & A(x, y), u_{2}, \ldots, u_{n} \|_{Y} \\
\quad & \lim _{j \rightarrow \infty} \frac{1}{2^{j}}\left\|D g\left(2^{j} x, 2^{j} y\right), u_{2}, \ldots, u_{n}\right\|_{Y} \\
& =\lim _{j \rightarrow \infty} \frac{1}{2^{j}}\left\|D f\left(2^{j+1} x, 2^{j+1} y\right)-8 D f\left(2^{j} x, 2^{j} y\right), u_{2}, \ldots, u_{n}\right\|_{Y} \\
& \leq \lim _{j \rightarrow \infty} \frac{1}{2^{j}}\left[\left\|D f\left(2^{j+1} x, 2^{j+1} y\right), u_{2}, \ldots, u_{n}\right\|_{Y}+8\left\|D f\left(2^{j} x, 2^{j} y\right), u_{2}, \ldots, u_{n}\right\|_{Y}\right] \\
& \leq \lim _{j \rightarrow \infty} \frac{1}{2^{j}}\left[\varphi\left(2^{j+1} x, 2^{j+1} y, u_{2}, \ldots, u_{n}\right)+8 \varphi\left(2^{j} x, 2^{j} y, u_{2}, \ldots, u_{n}\right)\right]=0
\end{array}
$$

for all $x, y, u_{2}, \ldots, u_{n} \in X$. By Lemma 1.6(3), $D A(x, y)=0$ for all $x, y \in X$. Hence, the mapping $A$ satisfies (1.1). By [11, Lemma 2.3], the mapping $x \rightarrow A(2 x)-8 A(x)$ is additive. Therefore, $A(2 x)=2 A(x)$ implies that the mapping $A$ is additive. 
To prove the uniqueness of $A$, let $B: X \rightarrow Y$ be another additive mapping satisfying (2.4). Fix $x \in X$. Clearly, $A\left(2^{l} x\right)=2^{l} A(x)$ and $B\left(2^{l} x\right)=2^{l} B(x)$ for all $l \in \mathbb{N}$. It follows from (2.4) that

$$
\begin{aligned}
\left\|A(x)-B(x), u_{2}, \ldots, u_{n}\right\|_{Y}= & \left\|\frac{A\left(2^{l} x\right)}{2^{l}}-\frac{B\left(2^{l} x\right)}{2^{l}}, u_{2}, \ldots, u_{n}\right\|_{Y} \\
\leq & \frac{1}{2^{l}}\left[\left\|f\left(2^{l+1} x\right)-8 f\left(2^{l} x\right)-A\left(2^{l} x\right), u_{2}, \ldots, u_{n}\right\|_{Y}\right. \\
& \left.+\left\|B\left(2^{l} x\right)-f\left(2^{l+1} x\right)+8 f\left(2^{l} x\right), u_{2}, \ldots, u_{n}\right\|_{Y}\right] \\
\leq & \frac{1}{2^{l}} \sum_{j=0}^{\infty} \frac{1}{2^{j}} \widetilde{\varphi}\left(2^{j+l} x, u_{2}, \ldots, u_{n}\right) \\
\leq & \sum_{j=0}^{\infty} \frac{1}{2^{j+l}} \tilde{\varphi}\left(2^{j+l} x, u_{2}, \ldots, u_{n}\right)=\sum_{j=l}^{\infty} \frac{1}{2^{j}} \widetilde{\varphi}\left(2^{j} x, u_{2}, \ldots, u_{n}\right)
\end{aligned}
$$

for all $x, u_{2}, \ldots, u_{n} \in X$, and $l \in \mathbb{N}$. By (2.2), we see that the right-hand side of the above inequality tends to 0 as $l \rightarrow \infty$. Therefore, $\left\|A(x)-B(x), u_{2}, \ldots, u_{n}\right\|_{Y}=0$ for all $u_{2}, \ldots, u_{n} \in X$. By Lemma 1.6, we can conclude that $A(x)=B(x)$ for all $x \in X$. So, $A=B$. This proves the uniqueness of $A$.

Theorem 2.2. Let $X$ be a linear space and $Y$ an $n$-Banach space. Let $f: X \rightarrow Y$ be a mapping with $f(0)=0$ for which there is a function $\varphi: X^{n+1} \rightarrow[0, \infty)$ such that

$$
\begin{gathered}
\sum_{j=1}^{\infty} 2^{j} \varphi\left(\frac{x}{2^{j}}, \frac{y}{2^{j}}, u_{2}, \ldots, u_{n}\right)<\infty, \\
\left\|D f(x, y), u_{2}, \ldots, u_{n}\right\|_{Y} \leq \varphi\left(x, y, u_{2}, \ldots, u_{n}\right)
\end{gathered}
$$

for all $x, y, u_{2}, \ldots, u_{n} \in X$. Then, there is a unique additive mapping $A: X \rightarrow Y$ such that

$$
\left\|f(2 x)-8 f(x)-A(x), u_{2}, \ldots, u_{n}\right\|_{Y} \leq \sum_{j=1}^{\infty} 2^{j-1} \tilde{\varphi}\left(\frac{x}{2^{j}}, u_{2}, \ldots, u_{n}\right)
$$

for all $x, u_{2}, \ldots, u_{n} \in X$, where $\tilde{\varphi}\left(x, u_{2}, \ldots, u_{n}\right)$ is defined as in Theorem 2.1 .

Proof. The proof is similar to the proof of Theorem 2.1.

Corollary 2.3. Let $X$ be a normed space and $Y$ an $n$-Banach space. Let $\theta \in[0, \infty), p, r_{2}, \ldots, r_{n} \in$ $(0, \infty)$ such that $p \neq 1$, and let $f: X \rightarrow Y$ be a mapping with $f(0)=0$ such that

$$
\left\|D f(x, y), u_{2}, \ldots, u_{n}\right\|_{Y} \leq \theta\left(\|x\|_{X}^{p}+\|y\|_{X}^{p}\right)\left\|u_{2}\right\|_{X}^{r_{2}} \cdots\left\|u_{n}\right\|_{X}^{r_{n}}
$$


for all $x, y, u_{2}, \ldots, u_{n} \in X$. Then, there exists a unique additive mapping $A: X \rightarrow Y$ such that

$$
\left\|f(2 x)-8 f(x)-A(x), u_{2}, \ldots, u_{n}\right\|_{Y} \leq \frac{\theta \epsilon\|x\|_{X}^{p}\left\|u_{2}\right\|_{X}^{r_{2}} \cdots\left\|u_{n}\right\|_{X}^{r_{n}}}{\left|\left(2-2^{p}\right)\left(k^{3}-k\right)\right|}
$$

for all $x, u_{2}, \ldots, u_{n} \in X$, where

$$
\begin{aligned}
\epsilon= & \left(1+|k|+2^{3-p}|k|\right)\left[(2 k+1)^{p}+(2 k-1)^{p}\right]+2|k|+13+3^{p}+3|k|^{p}+16 k^{2}+3^{p}|k|^{p}+2^{p+1} k^{2} \\
& +2^{p}\left(5+|k|^{p}\right)+2|k+1|^{p}+2|k-1|^{p}+2^{3-p}\left(2+|k|+|k|^{p}+3^{p}|k|^{p}\right)+\frac{(|k|+1)|k+1|^{p}}{|k-1|} \\
& +\frac{2^{3-p}|k|}{|k-1|}|k+1|^{p}+\left(1+8 k^{2}+2^{p} k^{2}\right)|k-1|^{p-1}+\frac{2^{p}|k|^{p}\left(k^{2}+|k|-1\right)}{|k-1|} \\
& +\frac{2}{|k-1|}+\frac{|k|\left(2^{3-p}+1\right)}{|k-1|}|3 k-1|^{p}+\frac{8 k^{2}+2|k|-8}{|k-1|}|k|^{p} .
\end{aligned}
$$

Proof. Define $\varphi(x, y)=\theta\left(\|x\|_{X}^{p}+\|y\|_{X}^{p}\right)\left\|u_{2}\right\|_{X}^{r_{2}} \cdots\left\|u_{n}\right\|_{X}^{r_{n}}$ for all $x, y, u_{2}, \ldots, u_{n} \in X$, and apply Theorems 2.1 and 2.2.

The following example shows that the assumption $p \neq 1$ cannot be omitted in Corollary 2.3.

Example 2.4. Let $X=\mathbb{C}$ be a linear space over $\mathbb{R}$. Define $\|\cdot, \cdot\|: X \times X \rightarrow \mathbb{R}$ by $\left\|x_{1}, x_{2}\right\|=$ $\left|a_{1} b_{2}-a_{2} b_{1}\right|$, where $x_{j}=a_{j}+b_{j} i \in \mathbb{C}, a_{j}, b_{j} \in \mathbb{R}, j=1,2(i=\sqrt{-1}$ is the imaginary unit). Then, $(X,\|\cdot, \cdot\|)$ is a 2 -normed linear space.

Let $\phi: \mathbb{C} \rightarrow \mathbb{C}$ defined by

$$
\phi(x)= \begin{cases}x, & \text { for }|x|<1 \\ 1, & \text { for }|x| \geq 1\end{cases}
$$

Consider the function $f: \mathbb{C} \rightarrow \mathbb{C}$ defined by

$$
f(x)=\sum_{m=0}^{\infty} \alpha^{-m} \phi\left(\alpha^{m} x\right)
$$

for all $x \in \mathbb{C}$, where $\alpha>|k|$. Then, $f$ satisfies the functional inequality

$$
\|D f(x, y), u\| \leq \frac{4 \alpha^{2}(|k|+1)}{\alpha-1}(|x|+|y|)|u|
$$

for all $x, y, u \in \mathbb{C}$, but there do not exist an additive mapping $A: \mathbb{C} \rightarrow \mathbb{C}$ and a constant $d>0$ such that $\|f(x)-A(x), u\| \leq d|x \| u|$ for all $x, u \in \mathbb{C}$. 
It is clear that $|f(x)| \leq \alpha /(\alpha-1)$ for all $x \in \mathbb{C}$. If $|x|+|y|=0$ or $|x|+|y| \geq 1 / \alpha$ for all $x, y \in \mathbb{C}$, then the inequality (2.47) holds. Now suppose that $0<|x|+|y|<1 / \alpha$. Then, there exists an integer $n \geq 1$ such that

$$
\frac{1}{\alpha^{n+1}} \leq|x|+|y|<\frac{1}{\alpha^{n}}
$$

Hence, $\alpha^{m}|k x \pm y|<1, \alpha^{m}|x \pm y|<1, \alpha^{m}|x|<1$ for all $m=0,1, \ldots, n-1$. From the definition of $f$ and (2.48), we obtain that

$$
\begin{array}{rl}
\| D & f(x, y), u \| \\
= & \| \sum_{m=n}^{\infty} \alpha^{-m} \phi\left(\alpha^{m}(k x+y)\right)+\sum_{m=n}^{\infty} \alpha^{-m} \phi\left(\alpha^{m}(k x-y)\right)-k \sum_{m=n}^{\infty} \alpha^{-m} \phi\left(\alpha^{m}(x+y)\right) \\
& -k \sum_{m=n}^{\infty} \alpha^{-m} \phi\left(\alpha^{m}(x-y)\right)-2 \sum_{m=n}^{\infty} \alpha^{-m} \phi\left(\alpha^{m} k x\right)+2 k \sum_{m=n}^{\infty} \alpha^{-m} \phi\left(\alpha^{m} x\right), u \| \\
\leq & \frac{4 \alpha^{2}(|k|+1)}{\alpha-1}(|x|+|y|)|u| .
\end{array}
$$

Therefore, $f$ satisfies (2.47). Now, we claim that the functional equation (1.1) is not stable for $p=1$ in Corollary 2.3. Suppose on the contrary that there exist an additive mapping $A: \mathbb{C} \rightarrow \mathbb{C}$ and a constant $d>0$ such that $\|f(x)-A(x), u\| \leq d|x \| u|$ for all $x, u \in \mathbb{C}$. Then, there exists a constant $c \in \mathbb{C}$ such that $A(x)=c x$ for all rational numbers $x$. So, we obtain that

$$
\|f(x), u\| \leq(d+|c|)|x \| u|
$$

for all rational numbers $x$ and all $u \in \mathbb{C}$. Let $s \in \mathbb{N}$ with $s+1>d+|c|$. If $x$ is a rational number in $\left(0, \alpha^{-s}\right)$ and $u=b i(b \in \mathbb{R})$, then $\alpha^{m} x \in(0,1)$ for all $m=0,1, \ldots, s$, and we get

$$
\|f(x), u\|=\left\|\sum_{m=0}^{\infty} \frac{\phi\left(\alpha^{m} x\right)}{\alpha^{m}}, u\right\| \geq \sum_{m=0}^{s} \frac{\phi\left(\alpha^{m} x\right)}{\alpha^{m}}|b|=(s+1) x|b|>(d+|c|) x|b|=(d+|c|)|x \| u|,
$$

which contradicts (2.50).

Theorem 2.5. Let $X$ be a linear space and $Y$ an n-Banach space. Let $f: X \rightarrow Y$ be a mapping with $f(0)=0$ for which there is a function $\varphi: X^{n+1} \rightarrow[0, \infty)$ such that

$$
\begin{gathered}
\sum_{j=0}^{\infty} \frac{1}{8^{j}} \varphi\left(2^{j} x, 2^{j} y, u_{2}, \ldots, u_{n}\right)<\infty, \\
\left\|D f(x, y), u_{2}, \ldots, u_{n}\right\|_{Y} \leq \varphi\left(x, y, u_{2}, \ldots, u_{n}\right)
\end{gathered}
$$


for all $x, y, u_{2}, \ldots, u_{n} \in X$. Then, there is a unique cubic mapping $C: X \rightarrow Y$ such that

$$
\left\|f(2 x)-2 f(x)-C(x), u_{2}, \ldots, u_{n}\right\|_{Y} \leq \sum_{j=0}^{\infty} \frac{1}{8^{j+1}} \tilde{\varphi}\left(2^{j} x, u_{2}, \ldots, u_{n}\right)
$$

for all $x, u_{2}, \ldots, u_{n} \in X$, where $\tilde{\varphi}\left(x, u_{2}, \ldots, u_{n}\right)$ is defined as in Theorem 2.1 .

Proof. As in the proof of Theorem 2.1, we have

$$
\left\|f(4 x)-10 f(2 x)+16 f(x), u_{2}, \ldots, u_{n}\right\|_{Y} \leq \tilde{\varphi}\left(x, u_{2}, \ldots, u_{n}\right)
$$

for all $x \in X$, where $\tilde{\varphi}\left(x, u_{2}, \ldots, u_{n}\right)$ is defined as in Theorem 2.1.

have

Now, let $h: X \rightarrow Y$ be the mapping defined by $h(x):=f(2 x)-2 f(x)$. By (2.55), we

$$
\left\|h(2 x)-8 h(x), u_{2}, \ldots, u_{n}\right\|_{Y} \leq \tilde{\varphi}\left(x, u_{2}, \ldots, u_{n}\right)
$$

for all $x \in X$. Replacing $x$ by $2^{j} x$ in (2.56) and dividing both sides of (2.56) by $8^{j+1}$, we get

$$
\left\|\frac{1}{8^{j}} h\left(2^{j} x\right)-\frac{1}{8^{j+1}} h\left(2^{j+1} x\right), u_{2}, \ldots, u_{n}\right\|_{Y} \leq \frac{1}{8^{j+1}} \tilde{\varphi}\left(2^{j} x, u_{2}, \ldots, u_{n}\right)
$$

for all $x, u_{2}, \ldots, u_{n} \in X$ and all integers $j \geq 0$. For all integers $l, m$ with $0 \leq l<m$, we have

$$
\begin{aligned}
\left\|\frac{1}{8^{l}} h\left(2^{l} x\right)-\frac{1}{8^{m}} h\left(2^{m} x\right), u_{2}, \ldots, u_{n}\right\|_{Y} & \leq \sum_{j=l}^{m-1}\left\|\frac{1}{8^{j}} h\left(2^{j} x\right)-\frac{1}{8^{j+1}} h\left(2^{j+1} x\right), u_{2}, \ldots, u_{n}\right\|_{Y} \\
& \leq \sum_{j=l}^{m-1} \frac{1}{8^{j+1}} \tilde{\varphi}\left(2^{j} x, u_{2}, \ldots, u_{n}\right)
\end{aligned}
$$

for all $x, u_{2}, \ldots, u_{n} \in X$. So, we get

$$
\lim _{l, m \rightarrow \infty}\left\|\frac{1}{8^{l}} h\left(2^{l} x\right)-\frac{1}{8^{m}} h\left(2^{m} x\right), u_{2}, \ldots, u_{n}\right\|_{Y}=0
$$

for all $x, u_{2}, \ldots, u_{n} \in X$. This shows that the sequence $\left\{\left(1 / 8^{j}\right) h\left(2^{j} x\right)\right\}$ is a Cauchy sequence in $Y$. Since $Y$ is an $n$-Banach space, the sequence $\left\{\left(1 / 8^{j}\right) h\left(2^{j} x\right)\right\}$ converges. So, we can define a mapping $C: X \rightarrow Y$ by

$$
C(x):=\lim _{j \rightarrow \infty} \frac{1}{8^{j}} h\left(2^{j} x\right)
$$


for all $x \in X$. Putting $l=0$, then passing the limit $m \rightarrow \infty$ in (2.58), and using Lemma 1.6(4), we get

$$
\left\|h(x)-C(x), u_{2}, \ldots, u_{n}\right\|_{Y} \leq \sum_{j=0}^{\infty} \frac{1}{8^{j+1}} \tilde{\varphi}\left(2^{j} x, u_{2}, \ldots, u_{n}\right)
$$

for all $x, u_{2}, \ldots, u_{n} \in X$.

Now we show that $C$ is cubic. By Lemma 1.6, (2.52), (2.58), and (2.60), we have

$$
\begin{aligned}
\left\|C(2 x)-8 C(x), u_{2}, \ldots, u_{n}\right\|_{Y} & =\lim _{j \rightarrow \infty}\left\|\frac{1}{8^{j}} h\left(2^{j+1} x\right)-\frac{1}{8^{j-1}} h\left(2^{j} x\right), u_{2}, \ldots, u_{n}\right\|_{Y} \\
& =8 \lim _{j \rightarrow \infty}\left\|\frac{1}{8^{j+1}} h\left(2^{j+1} x\right)-\frac{1}{8^{j}} h\left(2^{j} x\right), u_{2}, \ldots, u_{n}\right\|_{Y} \\
& \leq \lim _{j \rightarrow \infty} \frac{1}{8^{j}} \tilde{\varphi}\left(2^{j} x, u_{2}, \ldots, u_{n}\right)=0
\end{aligned}
$$

for all $x, u_{2}, \ldots, u_{n} \in X$. By Lemma 1.6(3), $C(2 x)=8 C(x)$ for all $x \in X$. Also, by Lemma 1.6(4), (2.52), (2.53), and (2.60), we get

$$
\begin{aligned}
& \left\|D C(x, y), u_{2}, \ldots, u_{n}\right\|_{Y} \\
& \quad=\lim _{j \rightarrow \infty} \frac{1}{8^{j}}\left\|D h\left(2^{j} x, 2^{j} y\right), u_{2}, \ldots, u_{n}\right\|_{Y} \\
& \quad=\lim _{j \rightarrow \infty} \frac{1}{8^{j}}\left\|D f\left(2^{j+1} x, 2^{j+1} y\right)-2 D f\left(2^{j} x, 2^{j} y\right), u_{2}, \ldots, u_{n}\right\|_{Y} \\
& \leq \lim _{j \rightarrow \infty} \frac{1}{8^{j}}\left[\left\|D f\left(2^{j+1} x, 2^{j+1} y\right), u_{2}, \ldots, u_{n}\right\|_{Y}+2\left\|D f\left(2^{j} x, 2^{j} y\right), u_{2}, \ldots, u_{n}\right\|_{Y}\right] \\
& \quad \leq \lim _{j \rightarrow \infty} \frac{1}{8^{j}}\left[\varphi\left(2^{j+1} x, 2^{j+1} y, u_{2}, \ldots, u_{n}\right)+2 \varphi\left(2^{j} x, 2^{j} y, u_{2}, \ldots, u_{n}\right)\right]=0
\end{aligned}
$$

for all $x, y, u_{2}, \ldots, u_{n} \in X$. By Lemma 1.6(3), $D C(x, y)=0$ for all $x, y \in X$. Hence the mapping $C$ satisfies (1.1). By [11, Lemma 2.3], the mapping $x \rightarrow C(2 x)-2 C(x)$ is cubic. Therefore, $C(2 x)=8 C(x)$ implies that the mapping $C$ is cubic. 
To prove the uniqueness of $C$, let $S: X \rightarrow Y$ be another cubic mapping satisfying (2.54). Fix $x \in X$. Clearly, $C\left(2^{l} x\right)=8^{l} A(x)$ and $S\left(2^{l} x\right)=8^{l} S(x)$ for all $l \in \mathbb{N}$. It follows from (2.54) that

$$
\begin{aligned}
\left\|C(x)-S(x), u_{2}, \ldots, u_{n}\right\|_{Y}= & \left\|\frac{C\left(2^{l} x\right)}{8^{l}}-\frac{S\left(2^{l} x\right)}{8^{l}}, u_{2}, \ldots, u_{n}\right\|_{Y} \\
\leq & \frac{1}{8^{l}}\left[\left\|f\left(2^{l+1} x\right)-2 f\left(2^{l} x\right)-C\left(2^{l} x\right), u_{2}, \ldots, u_{n}\right\|_{Y}\right. \\
& \left.+\left\|S\left(2^{l} x\right)-f\left(2^{l+1} x\right)+2 f\left(2^{l} x\right), u_{2}, \ldots, u_{n}\right\|_{Y}\right] \\
\leq & \frac{1}{8^{l}} \sum_{j=0}^{\infty} \frac{1}{8^{j}} \tilde{\varphi}\left(2^{j+l} x, u_{2}, \ldots, u_{n}\right) \\
\leq & \sum_{j=0}^{\infty} \frac{1}{8^{j+l}} \tilde{\varphi}\left(2^{j+l} x, u_{2}, \ldots, u_{n}\right)=\sum_{j=l}^{\infty} \frac{1}{8^{j}} \tilde{\varphi}\left(2^{j} x, u_{2}, \ldots, u_{n}\right)
\end{aligned}
$$

for all $x, u_{2}, \ldots, u_{n} \in X$, and $l \in \mathbb{N}$. By (2.52), we see that the right-hand side of the above inequality tends to 0 as $l \rightarrow \infty$. Therefore, $\left\|C(x)-S(x), u_{2}, \ldots, u_{n}\right\|_{Y}=0$ for all $u_{2}, \ldots, u_{n} \in X$. By Lemma 1.6, we can conclude that $C(x)=S(x)$ for all $x \in X$. So $C=S$. This proves the uniqueness of $C$.

Theorem 2.6. Let $X$ be a linear space and $Y$ an $n$-Banach space. Let $f: X \rightarrow Y$ be a mapping with $f(0)=0$ for which there is a function $\varphi: X^{n+1} \rightarrow[0, \infty)$ such that

$$
\begin{gathered}
\sum_{j=1}^{\infty} 8^{j} \varphi\left(\frac{x}{2^{j}}, \frac{y}{2^{j}}, u_{2}, \ldots, u_{n}\right)<\infty, \\
\left\|D f(x, y), u_{2}, \ldots, u_{n}\right\|_{Y} \leq \varphi\left(x, y, u_{2}, \ldots, u_{n}\right)
\end{gathered}
$$

for all $x, y, u_{2}, \ldots, u_{n} \in X$. Then, there is a unique cubic mapping $C: X \rightarrow Y$ such that

$$
\left\|f(2 x)-2 f(x)-C(x), u_{2}, \ldots, u_{n}\right\|_{Y} \leq \sum_{j=1}^{\infty} 8^{j-1} \tilde{\varphi}\left(\frac{x}{2^{j}}, u_{2}, \ldots, u_{n}\right)
$$

for all $x, u_{2}, \ldots, u_{n} \in X$, where $\tilde{\varphi}\left(x, u_{2}, \ldots, u_{n}\right)$ is defined as in Theorem 2.1 .

Proof. The proof is similar to the proof of Theorem 2.5.

Corollary 2.7. Let $X$ be a normed space and $Y$ an $n$-Banach space. Let $\theta \in[0, \infty), p, r_{2}, \ldots, r_{n} \in$ $(0, \infty)$ such that $p \neq 3$, and let $f: X \rightarrow Y$ be a mapping with $f(0)=0$ such that

$$
\left\|D f(x, y), u_{2}, \ldots, u_{n}\right\|_{Y} \leq \theta\left(\|x\|_{X}^{p}+\|y\|_{X}^{p}\right)\left\|u_{2}\right\|_{X}^{r_{2}} \cdots\left\|u_{n}\right\|_{X}^{r_{n}}
$$


for all $x, y, u_{2}, \ldots, u_{n} \in X$. Then, there exists a unique cubic mapping $C: X \rightarrow Y$ such that

$$
\left\|f(2 x)-2 f(x)-C(x), u_{2}, \ldots, u_{n}\right\|_{Y} \leq \frac{\theta \epsilon\|x\|_{X}^{p}\left\|u_{2}\right\|_{X}^{r_{2}} \cdots\left\|u_{n}\right\|_{X}^{r_{n}}}{\left|\left(8-2^{p}\right)\left(k^{3}-k\right)\right|}
$$

for all $x, u_{2}, \ldots, u_{n} \in X$, where $\epsilon$ is defined as in Corollary 2.3 .

Proof. Define $\varphi(x, y)=\theta\left(\|x\|_{X}^{p}+\|y\|_{X}^{p}\right)\left\|u_{2}\right\|_{X}^{r_{2}} \cdots\left\|u_{n}\right\|_{X}^{r_{n}}$ for all $x, y, u_{2}, \ldots, u_{n} \in X$, and apply Theorems 2.5 and 2.6.

The following example shows that the the generalized Hyers-Ulam stability problem for the case of $p=3$ was excluded in Corollary 2.7.

Example 2.8. Let $X=\mathbb{C}$ be a linear space over $\mathbb{R}$, and let $\|\cdot, \cdot\|: X \times X \rightarrow \mathbb{R}$ be defined as in Example 2.4. Then, $(X,\|\cdot, \cdot\|)$ is a 2-normed linear space.

Let $\phi: \mathbb{C} \rightarrow \mathbb{C}$ be defined by

$$
\phi(x)= \begin{cases}x^{3}, & \text { for }|x|<1 \\ 1, & \text { for }|x| \geq 1\end{cases}
$$

Consider the function $f: \mathbb{C} \rightarrow \mathbb{C}$ defined by

$$
f(x)=\sum_{m=0}^{\infty} \alpha^{-3 m} \phi\left(\alpha^{m} x\right)
$$

for all $x \in \mathbb{C}$, where $\alpha>|k|$. Then, $f$ satisfies the functional inequality

$$
\|D f(x, y), u\| \leq \frac{4 \alpha^{6}(|k|+1)}{\alpha^{3}-1}\left(|x|^{3}+|y|^{3}\right)|u|
$$

for all $x, y, u \in \mathbb{C}$, but there do not exist a cubic mapping $C: \mathbb{C} \rightarrow \mathbb{C}$ and a constant $d>0$ such that $\|f(x)-C(x), u\| \leq d|x|^{3}|u|$ for all $x, u \in \mathbb{C}$.

It is clear that $|f(x)| \leq \alpha^{3} /\left(\alpha^{3}-1\right)$ for all $x \in \mathbb{C}$. If $|x|^{3}+|y|^{3}=0$ or $|x|^{3}+|y|^{3} \geq 1 / \alpha^{3}$ for all $x, y \in \mathbb{C}$, then inequality (2.71) holds. Now suppose that $0<|x|^{3}+|y|^{3}<1 / \alpha^{3}$. Then, there exists an integer $n \geq 1$ such that

$$
\frac{1}{\alpha^{3(n+1)}} \leq|x|^{3}+|y|^{3}<\frac{1}{\alpha^{3 n}}
$$


Hence, $\alpha^{m}|k x \pm y|<1, \alpha^{m}|x \pm y|<1, \alpha^{m}|x|<1$ for all $m=0,1, \ldots, n-1$. From the definition of $f$ and (2.72), we obtain that

$$
\begin{aligned}
\|D f(x, y), u\|= & \| \sum_{m=n}^{\infty} \alpha^{-3 m} \phi\left(\alpha^{m}(k x+y)\right)+\sum_{m=n}^{\infty} \alpha^{-3 m} \phi\left(\alpha^{m}(k x-y)\right)-k \sum_{m=n}^{\infty} \alpha^{-3 m} \phi\left(\alpha^{m}(x+y)\right) \\
& \quad-k \sum_{m=n}^{\infty} \alpha^{-3 m} \phi\left(\alpha^{m}(x-y)\right)-2 \sum_{m=n}^{\infty} \alpha^{-3 m} \phi\left(\alpha^{m} k x\right)+2 k \sum_{m=n}^{\infty} \alpha^{-3 m} \phi\left(\alpha^{m} x\right), u \| \\
\leq & \frac{4 \alpha^{6}(|k|+1)}{\alpha^{3}-1}\left(|x|^{3}+|y|^{3}\right)|u| .
\end{aligned}
$$

Therefore, $f$ satisfies (2.71). Now, we claim that the functional equation (1.1) is not stable for $p=3$ in Corollary 2.7. Suppose on the contrary that there exist a cubic mapping $C: \mathbb{C} \rightarrow \mathbb{C}$ and a constant $d>0$ such that $\|f(x)-C(x), u\| \leq d|x|^{3}|u|$ for all $x, u \in \mathbb{C}$. Then, there exists a constant $\beta \in \mathbb{C}$ such that $C(x)=\beta x^{3}$ for all rational numbers $x$. So, we obtain that

$$
\|f(x), u\| \leq(d+|\beta|)|x|^{3}|u|
$$

for all rational numbers $x$ and all $u \in \mathbb{C}$. Let $s \in \mathbb{N}$ with $s+1>d+|\beta|$. If $x$ is a rational number in $\left(0, \alpha^{-s}\right)$ and $u=b i(b \in \mathbb{R})$, then $\alpha^{m} x \in(0,1)$ for all $m=0,1, \ldots, s$, and we get

$$
\begin{aligned}
\|f(x), u\| & =\left\|\sum_{m=0}^{\infty} \frac{\phi\left(\alpha^{m} x\right)}{\alpha^{3 m}}, u\right\| \geq \sum_{m=0}^{s} \frac{\phi\left(\alpha^{m} x\right)}{\alpha^{3 m}}|b| \\
& =(s+1) x^{3}|b|>(d+|\beta|) x^{3}|b|=(d+|\beta|)|x|^{3}|u|,
\end{aligned}
$$

which contradicts (2.74).

Theorem 2.9. Let $X$ be a linear space and $Y$ an $n$-Banach space. Let $f: X \rightarrow Y$ be a mapping with $f(0)=0$ for which there is a function $\varphi: X^{n+1} \rightarrow[0, \infty)$ such that

$$
\begin{gathered}
\sum_{j=0}^{\infty} \frac{1}{2^{j}} \varphi\left(2^{j} x, 2^{j} y, u_{2}, \ldots, u_{n}\right)<\infty, \\
\left\|D f(x, y), u_{2}, \ldots, u_{n}\right\|_{Y} \leq \varphi\left(x, y, u_{2}, \ldots, u_{n}\right)
\end{gathered}
$$

for all $x, y, u_{2}, \ldots, u_{n} \in X$. Then, there exist a unique additive mapping $A: X \rightarrow Y$ and a unique cubic mapping $C: X \rightarrow Y$ such that

$$
\left\|f(x)-A(x)-C(x), u_{2}, \ldots, u_{n}\right\|_{Y} \leq \frac{1}{6} \sum_{j=0}^{\infty}\left(\frac{1}{2^{j+1}}+\frac{1}{8^{j+1}}\right) \tilde{\varphi}\left(2^{j} x, u_{2}, \ldots, u_{n}\right)
$$

for all $x, u_{2}, \ldots, u_{n} \in X$, where $\tilde{\varphi}\left(x, u_{2}, \ldots, u_{n}\right)$ is defined as in Theorem 2.1 . 
Proof. By Theorems 2.1 and 2.5, there exist an additive mapping $A^{\prime}: X \rightarrow Y$ and a cubic mapping $C^{\prime}: X \rightarrow Y$ such that

$$
\begin{aligned}
& \left\|f(2 x)-8 f(x)-A^{\prime}(x), u_{2}, \ldots, u_{n}\right\|_{Y} \leq \sum_{j=0}^{\infty} \frac{1}{2^{j+1}} \tilde{\varphi}\left(2^{j} x, u_{2}, \ldots, u_{n}\right), \\
& \left\|f(2 x)-2 f(x)-C^{\prime}(x), u_{2}, \ldots, u_{n}\right\|_{Y} \leq \sum_{j=0}^{\infty} \frac{1}{8^{j+1}} \tilde{\varphi}\left(2^{j} x, u_{2}, \ldots, u_{n}\right)
\end{aligned}
$$

for all $x, u_{2}, \ldots, u_{n} \in X$. Hence,

$$
\left\|f(x)+\frac{1}{6} A^{\prime}(x)-\frac{1}{6} C^{\prime}(x), u_{2}, \ldots, u_{n}\right\|_{Y} \leq \frac{1}{6} \sum_{j=0}^{\infty}\left(\frac{1}{2^{j+1}}+\frac{1}{8^{j+1}}\right) \tilde{\varphi}\left(2^{j} x, u_{2}, \ldots, u_{n}\right)
$$

for all $x \in X$. So, we obtain (2.78) by letting $A(x)=-(1 / 6) A^{\prime}(x)$ and $C(x)=(1 / 6) C^{\prime}(x)$ for all $x \in X$.

To prove the uniqueness of $A$ and $C$, let $A^{\prime \prime}, C^{\prime \prime}: X \rightarrow Y$ be another additive and cubic mapping satisfying (2.78). Fix $x \in X$. Let $A_{1}=A-A^{\prime \prime}$ and $C_{1}=C-C^{\prime \prime}$. So,

$$
\begin{aligned}
& \left\|A_{1}(x)+C_{1}(x), u_{2}, \ldots, u_{n}\right\|_{Y} \\
& \quad \leq\left\|f(x)-A(x)-C(x), u_{2}, \ldots, u_{n}\right\|_{Y}+\left\|f(x)-A^{\prime \prime}(x)-C^{\prime \prime}(x), u_{2}, \ldots, u_{n}\right\|_{Y} \\
& \quad \leq \frac{1}{3} \sum_{j=0}^{\infty}\left(\frac{1}{2^{j+1}}+\frac{1}{8^{j+1}}\right) \tilde{\varphi}\left(2^{j} x, u_{2}, \ldots, u_{n}\right)
\end{aligned}
$$

for all $x, u_{2}, \ldots, u_{n} \in X$. Then (2.76) implies that

$$
\lim _{n \rightarrow \infty} \frac{1}{8^{n}}\left\|A_{1}\left(2^{n} x\right)+C_{1}\left(2^{n} x\right), u_{2}, \ldots, u_{n}\right\|_{Y}=0
$$

for all $x, u_{2}, \ldots, u_{n} \in X$. Thus, $C_{1}=0$. So, it follows from (2.81) that

$$
\left\|A_{1}(x), u_{2}, \ldots, u_{n}\right\|_{Y} \leq \frac{1}{3} \sum_{j=0}^{\infty}\left(\frac{1}{2^{j+1}}+\frac{1}{8^{j+1}}\right) \tilde{\varphi}\left(2^{j} x, u_{2}, \ldots, u_{n}\right)
$$

for all $u_{2}, \ldots, u_{n} \in X$. Therefore, $A_{1}=0$.

Similarly to Theorem 2.9 , one can prove the following result. 
Theorem 2.10. Let $X$ be a linear space and $Y$ an n-Banach space. Let $f: X \rightarrow Y$ be a mapping with $f(0)=0$ for which there is a function $\varphi: X^{n+1} \rightarrow[0, \infty)$ such that

$$
\begin{gathered}
\sum_{j=0}^{\infty} 8^{j} \varphi\left(\frac{x}{2^{j}}, \frac{y}{2^{j}}, u_{2}, \ldots, u_{n}\right)<\infty, \\
\left\|D f(x, y), u_{2}, \ldots, u_{n}\right\|_{Y} \leq \varphi\left(x, y, u_{2}, \ldots, u_{n}\right)
\end{gathered}
$$

for all $x, y, u_{2}, \ldots, u_{n} \in X$. Then, there exist a unique additive mapping $A: X \rightarrow Y$ and a unique cubic mapping $C: X \rightarrow Y$ such that

$$
\left\|f(x)-A(x)-C(x), u_{2}, \ldots, u_{n}\right\|_{Y} \leq \frac{1}{6} \sum_{j=1}^{\infty}\left(2^{j-1}+8^{j-1}\right) \tilde{\varphi}\left(\frac{x}{2^{j}}, u_{2}, \ldots, u_{n}\right)
$$

for all $x, u_{2}, \ldots, u_{n} \in X$, where $\tilde{\varphi}\left(x, u_{2}, \ldots, u_{n}\right)$ is defined as in Theorem 2.1.

Proof. The proof is similar to the proof of Theorem 2.9 and the result follows from Theorems 2.2 and 2.6.

Theorem 2.11. Let $X$ be a linear space and $Y$ an n-Banach space. Let $f: X \rightarrow Y$ be a mapping with $f(0)=0$ for which there is a function $\varphi: X^{n+1} \rightarrow[0, \infty)$ such that

$$
\begin{gathered}
\sum_{j=1}^{\infty} 2^{j} \varphi\left(\frac{x}{2^{j}}, \frac{y}{2^{j}}, u_{2}, \ldots, u_{n}\right)<\infty, \sum_{j=0}^{\infty} \frac{1}{8^{j}} \varphi\left(2^{j} x, 2^{j} y, u_{2}, \ldots, u_{n}\right)<\infty \\
\left\|D f(x, y), u_{2}, \ldots, u_{n}\right\|_{Y} \leq \varphi\left(x, y, u_{2}, \ldots, u_{n}\right)
\end{gathered}
$$

for all $x, y, u_{2}, \ldots, u_{n} \in X$. Then, there exist a unique additive mapping $A: X \rightarrow Y$ and a unique cubic mapping $C: X \rightarrow Y$ such that

$$
\begin{aligned}
& \left\|f(x)-A(x)-C(x), u_{2}, \ldots, u_{n}\right\|_{Y} \\
& \quad \leq \frac{1}{6}\left[\sum_{j=1}^{\infty} 2^{j-1} \tilde{\varphi}\left(\frac{x}{2^{j}}, u_{2}, \ldots, u_{n}\right)+\sum_{j=0}^{\infty} \frac{1}{8^{j+1}} \tilde{\varphi}\left(2^{j} x, u_{2}, \ldots, u_{n}\right)\right]
\end{aligned}
$$

for all $x, u_{2}, \ldots, u_{n} \in X$, where $\tilde{\varphi}\left(x, u_{2}, \ldots, u_{n}\right)$ is defined as in Theorem 2.1 .

Proof. The proof is similar to the proof of Theorem 2.9 and the result follows from Theorems 2.2 and 2.5 .

Corollary 2.12. Let $X$ be a normed space and $Y$ an $n$-Banach space. Let $\theta \in[0, \infty), r_{2}, \ldots, r_{n} \in$ $(0, \infty), p \in(0,1) \cup(1,3) \cup(3, \infty)$, and let $f: X \rightarrow Y$ be a mapping with $f(0)=0$ such that

$$
\left\|D f(x, y), u_{2}, \ldots, u_{n}\right\|_{Y} \leq \theta\left(\|x\|_{X}^{p}+\|y\|_{X}^{p}\right)\left\|u_{2}\right\|_{X}^{r_{2}} \cdots\left\|u_{n}\right\|_{X}^{r_{n}}
$$


for all $x, y, u_{2}, \ldots, u_{n} \in X$. Then, there exist a unique additive mapping $A: X \rightarrow Y$ and a unique cubic mapping $C: X \rightarrow Y$ such that

$$
\left\|f(x)-A(x)-C(x), u_{2}, \ldots, u_{n}\right\|_{Y} \leq \frac{1}{6\left|k^{3}-k\right|}\left(\frac{1}{\left|2-2^{p}\right|}+\frac{1}{\left|8-2^{p}\right|}\right) \theta \epsilon\|x\|_{X}^{p}\left\|u_{2}\right\|_{X}^{r_{2}} \cdots\left\|u_{n}\right\|_{X}^{r_{n}}
$$

for all $x, u_{2}, \ldots, u_{n} \in X$, where $\epsilon$ is defined as in Corollary 2.3 .

Proof. Define $\varphi(x, y)=\theta\left(\|x\|_{X}^{p}+\|y\|_{X}^{p}\right)\left\|u_{2}\right\|_{X}^{r_{2}} \cdots\left\|u_{n}\right\|_{X}^{r_{n}}$ for all $x, y, u_{2}, \ldots, u_{n} \in X$, and apply Theorems 2.9-2.11.

Remark 2.13. The generalized Hyers-Ulam stability problem for the cases of $p=1$ and $p=3$ was excluded in Corollary 2.12 (see Examples 2.4 and 2.8).

\section{Acknowledgments}

The authors would like to thank the Editor Professor Krzysztof Cieplinski and anonymous referees for their valuable comments and suggestions. The first author was supported by the National Natural Science Foundation of China (NNSFC) (grant No. 11171022).

\section{References}

[1] Z. Moszner, "On the stability of functional equations," Aequationes Mathematicae, vol. 77, no. 1-2, pp. 33-88, 2009.

[2] S. M. Ulam, A Collection of Mathematical Problems, vol. 8 of Interscience Tracts in Pure and Applied Mathematics, Interscience, New York, NY, USA, 1960.

[3] D. H. Hyers, "On the stability of the linear functional equation," Proceedings of the National Academy of Sciences of the United States of America, vol. 27, pp. 222-224, 1941.

[4] T. Aoki, "On the stability of the linear transformation in Banach spaces," Journal of the Mathematical Society of Japan, vol. 2, pp. 64-66, 1950.

[5] T. M. Rassias, "On the stability of the linear mapping in Banach spaces," Proceedings of the American Mathematical Society, vol. 72, no. 2, pp. 297-300, 1978.

[6] P. Găvruţa, "A generalization of the Hyers-Ulam-Rassias stability of approximately additive mappings," Journal of Mathematical Analysis and Applications, vol. 184, no. 3, pp. 431-436, 1994.

[7] R. P. Agarwal, B. Xu, and W. Zhang, "Stability of functional equations in single variable," Journal of Mathematical Analysis and Applications, vol. 288, no. 2, pp. 852-869, 2003.

[8] A. Najati and G. Z. Eskandani, "Stability of a mixed additive and cubic functional equation in quasiBanach spaces," Journal of Mathematical Analysis and Applications, vol. 342, no. 2, pp. 1318-1331, 2008.

[9] W.-G. Park, "Approximate additive mappings in 2-Banach spaces and related topics," Journal of Mathematical Analysis and Applications, vol. 376, no. 1, pp. 193-202, 2011.

[10] R. Saadati, Y. J. Cho, and J. Vahidi, "The stability of the quartic functional equation in various spaces," Computers E Mathematics with Applications, vol. 60, no. 7, pp. 1994-2002, 2010.

[11] T. Z. Xu, J. M. Rassias, and W. X. Xu, "Generalized Hyers-Ulam stability of a general mixed additivecubic functional equation in quasi-Banach spaces," Acta Mathematica Sinica, English Series, vol. 28, no. 3, pp. 529-560, 2011.

[12] T. Z. Xu, J. M. Rassias, and W. X. Xu, "Stability of a general mixed additive-cubic functional equation in non-Archimedean fuzzy normed spaces," Journal of Mathematical Physics, vol. 51, no. 9, Article ID 093508, 19 pages, 2010.

[13] S. Gähler, "2-metrische Räume und ihre topologische Struktur,” Mathematische Nachrichten, vol. 26, pp. 115-148, 1963. 
[14] S. Gähler, "Lineare 2-normierte Räume," Mathematische Nachrichten, vol. 28, pp. 1-43, 1964.

[15] Y. J. Cho, P. C. S. Lin, S. S. Kim, and A. Misiak, Theory of 2-Inner Product Spaces, Nova Science, Huntington, NY, USA, 2001.

[16] A. Misiak, "n-inner product spaces," Mathematische Nachrichten, vol. 140, pp. 299-319, 1989.

[17] X. Y. Chen and M. M. Song, "Characterizations on isometries in linear n-normed spaces," Nonlinear Analysis, vol. 72, no. 3-4, pp. 1895-1901, 2010.

[18] S. Gähler, “Über 2-Banach-Räume,” Mathematische Nachrichten, vol. 42, pp. 335-347, 1969.

[19] A. G. White, Jr., "2-Banach spaces," Mathematische Nachrichten, vol. 42, pp. 43-60, 1969. 


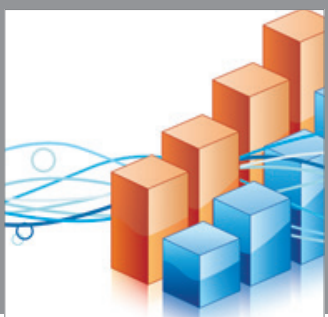

Advances in

Operations Research

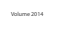

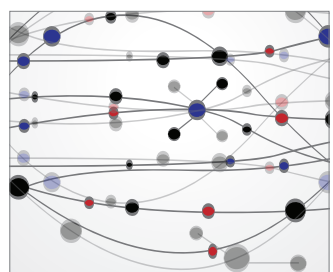

\section{The Scientific} World Journal
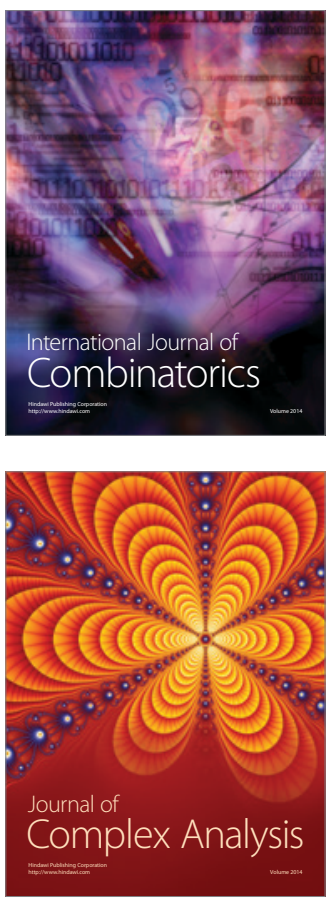

International Journal of

Mathematics and

Mathematical

Sciences
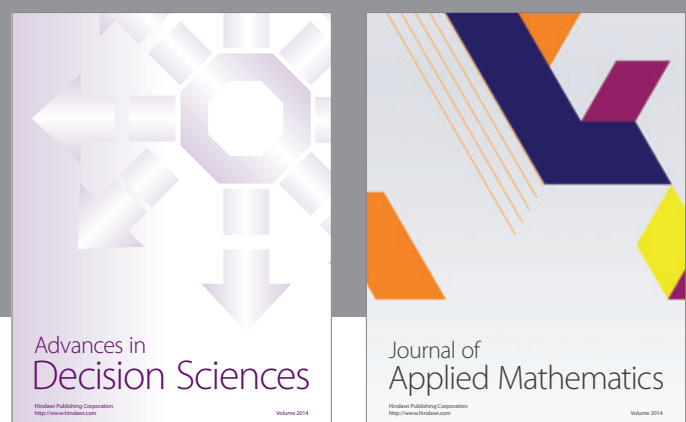

Journal of

Applied Mathematics
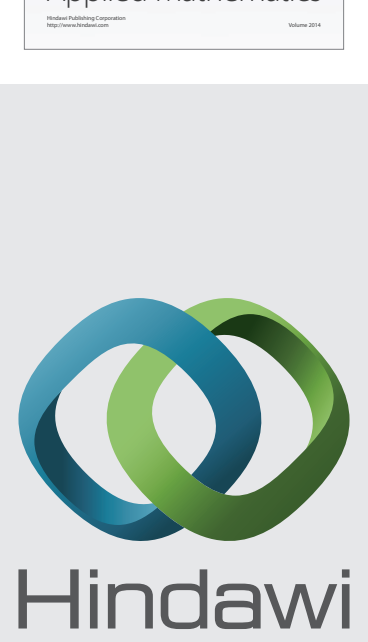

Submit your manuscripts at http://www.hindawi.com
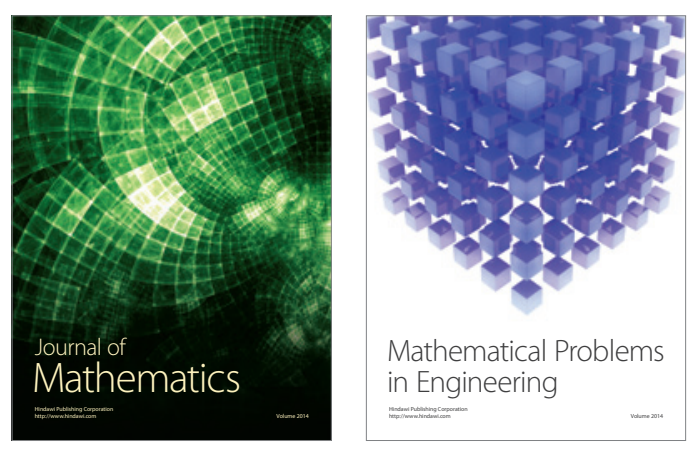

Mathematical Problems in Engineering
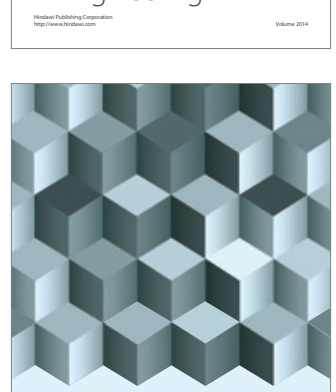

Journal of

Function Spaces
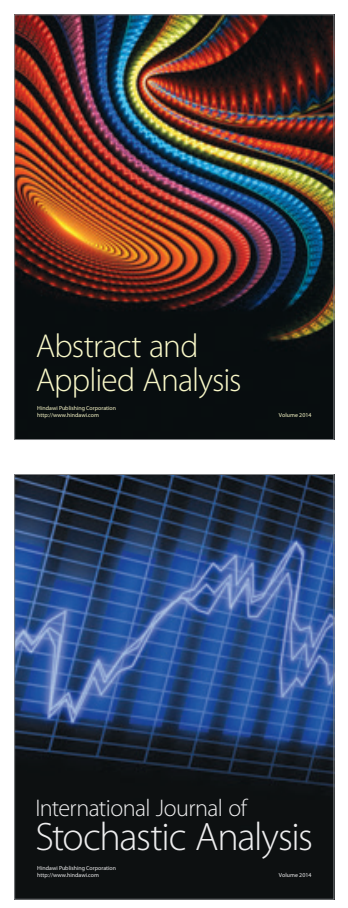

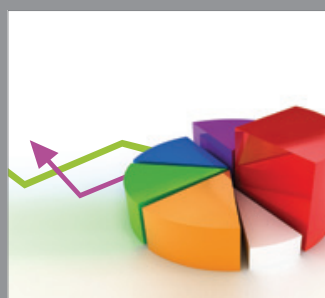

ournal of

Probability and Statistics

Promensencen
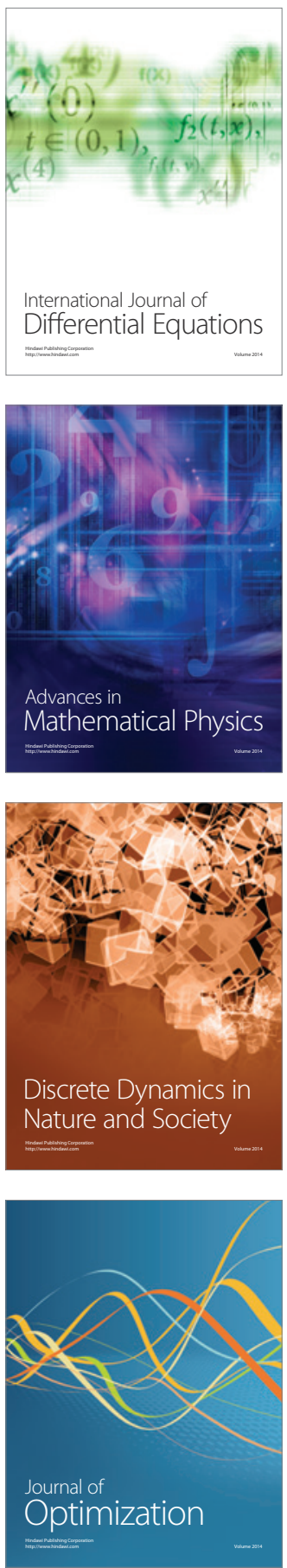